\title{
A Web User Interface of the Security Requirement Management Database Based on ISO/IEC 15408
}

\author{
Daisuke Horie, Shoichi Morimoto, and Jingde Cheng \\ Department of Information and Computer Sciences, \\ Saitama University, Saitama, 338-8570, Japan \\ \{horie, morimo, cheng\}@aise.ics.saitama-u.ac.jp
}

\begin{abstract}
In order to support design and development of secure information systems, we have proposed a security requirement management database based on the international standard ISO/IEC 15408. Design and development of secure information systems concern issues of information security engineering as well as software engineering. Our security requirement management database will be useful in practices only if we can provide its users with a highly usable user interface. This paper presents the design and development of a web user interface of our security requirement management database. We analyze and define usability requirements that the database should satisfy, present design and implementation of the web user interface, and show some examples for evaluating the interface from the viewpoint of usability engineering.
\end{abstract}

\section{Introduction}

Nowadays, security is an important factor in information system development. There is rising demand for security in information systems. Database technologies have successfully been applied to software engineering $[9,6,3$. Similarly, we can apply database technologies to information security engineering. Therefore, we have proposed a concept for a database to support design and development of secure information systems, named "ISEDS (Information Security Engineering Database System) 7]." ISEDS can collect and manage the past knowledge and experience for secure system design.

However, we had not sufficiently discussed how to use ISEDS yet. In order to achieve the purpose of ISEDS, it is necessary to clarify usage of ISEDS and usability requirements for ISEDS from the viewpoint of usability engineering. Moreover, it is desirable to implement a useful and convenient user interface which satisfies the requirements. In this paper, we analyze and define the requirements and present design and implementation of a web user interface. Users of ISEDS can easily and effectively use it for ISEDS anytime and anywhere by web browsers. 


\section{The Outline of ISEDS}

We developed ISEDS based on the international standard ISO/IEC 15408, because ISO/IEC 15408 defines common criteria that should be applied to validate a secure information system [5. ISEDS can store the knowledge or experience in security specifications for secure system design according to the common criteria. The data can be a guideline to design and development of secure information systems.

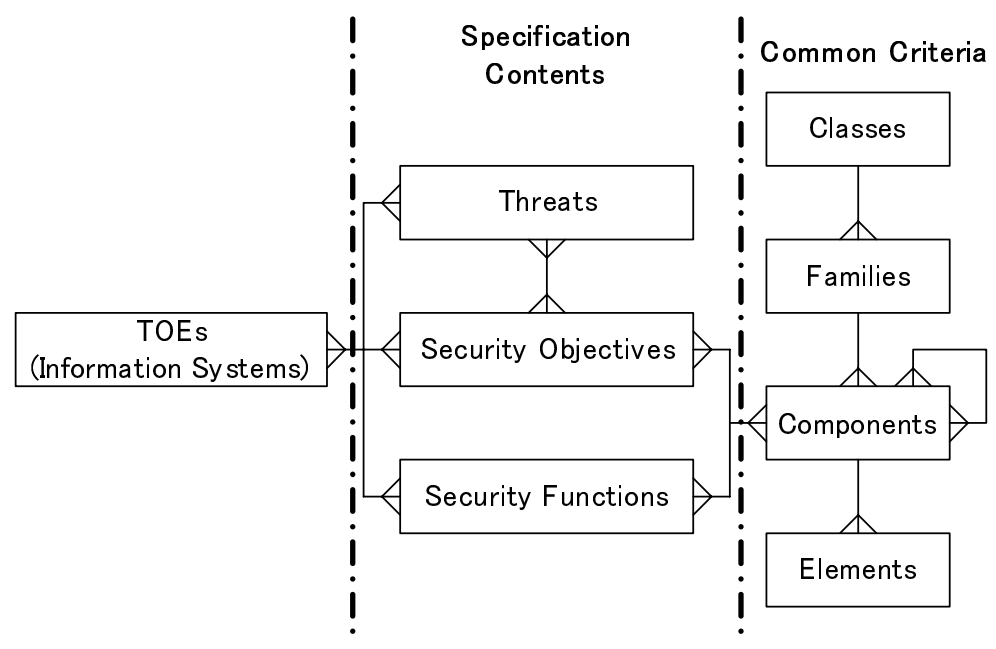

Fig. 1. The entity-relationship diagram of ISEDS

ISEDS has the structure as shown in Fig. 1. It is the entity-relationship diagram in relational notation [2]. Below, an italic word denotes a schema or its attribute in Fig. 1.

Applicants who apply to obtain the evaluation of ISO/IEC 15408 have to make and submit the specification document called 'security target' or 'protection profile' to the evaluation organization. A security target, ST for short, must describe the range of the target system which is evaluated (target of evaluation, TOE for short). A protection profile, PP for short, is a template for an ST. The schema TOE has attributes that are written to an ST or a PP, i.e., TOE Name, TOE Abstract, Author, Small Classification, Middle Classification, and Large Classification. The classifications denote TOE kinds. So far, Large Classification is only 'IT products.' Middle Classification is 'software,' 'hardware,' 'middleware,' and so on. Small Classification shows the concrete TOE kind, e.g., 'Database,' 'Firewall,' 'IC card,' 'OS,' 'Copier,' and so on. In addition to these attributes, we defined Document Type and Document File. Document Type is a flag for distinguishing an ST or a PP. Document File is an attribute for storing the binary file of the ST or PP. 
An ST or a PP must describe threats assumed in its category. Next, the documents must describe security objectives which oppose these threats. Thirdly, the documents must describe security criteria required for achievement of these objectives. In particular, the required security criteria must be quoted from the security functional requirements which are defined in ISO/IEC 15408 Part 2. For the implementation of security functional requirements, the documents must also describe that they are actually implemented as what functions in systems. These functions are called a TOE security function, TSF for short. TOE relates to one or more Threats, Security Objectives, and Security Functions. The schemata Threat, Security Objective, and Security Function have their ID numbers, abbreviation names, formal names, texts of the definition on the documents, and foreign keys to TOE. ISEDS can store such data of the documents.

Security functional requirements of Part 2 have a hierarchical structure which is composed by classes, families, components, and elements. Each element is an indivisible security requirement, and describes a security functional requirement exactly and directly. A component is a group of elements, and may be mutually dependent on the other components. A family is a group of components, and a class is a group of families. The schemata of security functional requirements have the attributes defined in ISO/IEC 15408 Part 2. ISEDS can also store such data of the criteria.

\section{The Web User Interface of ISEDS}

ISEDS can achieve its purpose only if we can provide its users with a usable user interface. Thus, we examine requirements to ISEDS from the viewpoint of software engineering, information security engineering, and usability engineering. In compliance with the requirements we have implemented the prototype of the user interface.

\subsection{Requirements for ISEDS}

We herein estimate and enumerate requirements for ISEDS.

1. From the viewpoint of software engineering, ISEDS must structurally collect and manage data of knowledge or experience for secure system design.

Requirement 1-1: Users must be able to structurally refer to the data using ISEDS.

Requirement 1-2: Users must be able to update the data using ISEDS.

2. From the viewpoint of information security engineering, ISEDS must provide useful information for secure system design and development.

Requirement 2-1: Users must be able to know what systems comply with ISO/IEC 15408 using ISEDS.

Users may refer to what TOEs exist. That is, the users may want to search whether or not the TOEs in firewall category exist. Moreover, users may refer to what threats are assumed in a category and what security objectives, security 
functions, or criteria of ISO/IEC 15408 are required in a category. For example, the users may refer to what threats are assumed in the TOEs of firewall category.

Requirement 2-2: Users must be able to know what security criteria are in ISO/IEC 15408 using ISEDS.

Users may refer to what criteria exist. The users may refer to what criteria about user data protection are. Moreover, users may refer to what categories, threats, or objectives require a criterion. The users may refer what systems require the criterion FTA_TSE.1. In addition, users may refer to hierarchical structure of ISO/IEC 15408. The users may refer to which criteria are included in the class FTA.

Requirement 2-3: Users must be able to know what threats are in the past systems using ISEDS.

Users may refer to what threats exist. The users may want to search whether or not the threats about spoofing exist. Moreover, users may refer to what categories assume a threat and what security objectives, security functions, or criteria can resist a threat. The user may refer to what security functions can resist spoofing.

Requirement 2-4: Users must be able to know what security countermeasures are in the past systems using ISEDS.

Users may refer to what security objectives exist. The users may want to search whether or not security objectives about concealment of IP addresses exist. Moreover, users may refer to what categories or threats require an objective and what security criteria are required for achievement of an objective. The users may refer to what security criteria are required for concealment of IP addresses.

At least, ISEDS should satisfy the above requirements. In order to provide useful functions satisfying the requirements, it is necessary to consider usability engineering. ISO 9241-11 defines that usability is extent to which a product can be used by specified users to achieve specified goals with effectiveness, efficiency and satisfaction in a specified context of use [4. The usability consists of four elements, i.e., effectiveness, efficiency, satisfaction, and context of use. The effectiveness is accuracy and completeness with which users achieve specified goals. The efficiency shows resources expended in relation to the accuracy and completeness with which users achieve goals. The satisfaction is freedom from discomfort, and positive attitudes towards the use of the product. The context of use shows users, tasks, equipment (hardware, software and materials), and the physical and social environments in which a product is used. ISEDS's interface should also satisfy these requirements for usability.

\subsection{Functions Provided by the Web User Interface}

We have implemented a prototype user interface satisfying the above requirements. The user interface provides the following functions. 
Function 1: Certified System's Specifications Search; the function to refer to all or specific data of certified system specifications. This function satisfies the requirements 1-1, 2-1, 2-3, and 2-4.

Function 2: Common Criteria Search; the function to refer to all or specific security criteria in ISO/IEC 15408. This function satisfies the requirements 1-1 and $2-2$.

Function 3: Free Word Search; the function to freely retrieve data in ISEDS. This function satisfies the efficiency and satisfaction.

Function 4: Update Function; the function to update the data in ISEDS. This function satisfies the requirement 1-2.

The effectiveness is satisfied by all functions. Moreover, we implemented the user interface using web technologies so as to satisfy the context of use, i.e., the requirement for 'anytime and anywhere.' The user interface is implemented with PHP, because it can dynamically generate web pages and has high affinity with PostgreSQL which implements ISEDS. The user interface generates SQL sentences based on user's requests and sends the SQL sentences to ISEDS. Next, the interface receives data from ISEDS and legibly displays the results. Users who are not well informed about SQL can easily and ubiquitously use ISEDS with web-browsers. Thus, the user interface satisfies the context of use.

\subsection{Examples}

We show some examples using the user interface.

\begin{tabular}{|l|l|}
\hline $\begin{array}{l}\text { Certified System's } \\
\text { Specifications Search }\end{array}$ & $\begin{array}{l}\text { Display TOE list } \\
\text { Display Threat list } \\
\text { Display Security Objective list } \\
\text { Display Security Function list }\end{array}$ \\
\hline Common Criteria Search & $\begin{array}{l}\text { Display Element list } \\
\text { Display Component list }\end{array}$ \\
$\begin{array}{ll}\text { Display Family list } \\
\text { Display Class list }\end{array}$ \\
\hline Free Word Search & \\
\hline Update Function & \\
\hline
\end{tabular}

Fig. 2. The top page of the user interface

Example 1: If users require retrieval such as the requirements 2-1, 2-3 or 2-4;

Click the required link from Certified System's Specifications Search menus in Fig. 2. All of the data corresponding to the selected menu will be displayed as Fig. 3. Moreover, one can narrow the range to input a specific keyword into the text box, select the radio button of the attribute including the specific keyword, and push the button 'search more.' For example, click the link 'TOE List.' All data of TOEs will be displayed. Input a keyword 'firewall' into the text box, 


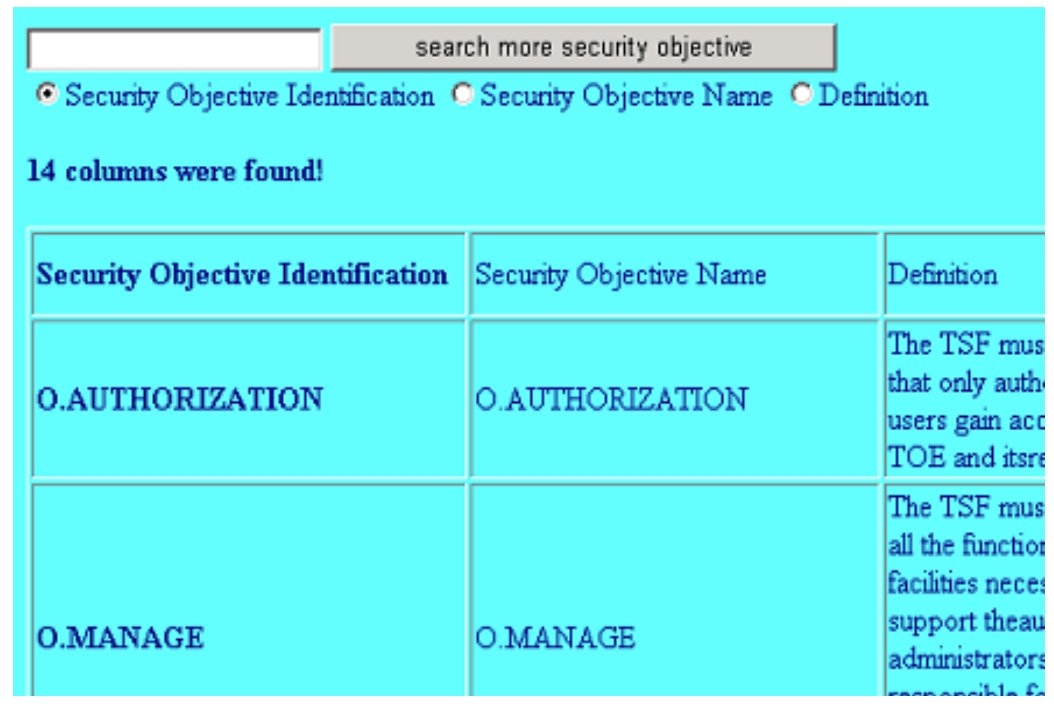

Fig. 3. The result display in the user interface

select the radio button 'Small Classification,' and push the button 'search more TOEs.' Then, data of documents in the firewall category will be displayed.

Example 2: If users require such as the requirement 2-2;

Click the required link from Common Criteria Search menus in Fig. 2. All of the data corresponding to the selected menu will be displayed as Fig. 3. Moreover, one can narrow the range as well as the example 1. For example, click the link 'Element List.' All data of elements displayed. Input a keyword 'recording' into the text box, select the radio button 'Definition,' and push the button 'search more elements.' Then, data of elements including the keyword 'recording' in the attribute 'Definition' will be displayed.

Example 3: If users require further retrieval;

Click the link 'Free Word Search' in Fig. 2. Users can freely choose the items to display and to narrow retrieval range. For example, click the link 'Free Word Search,' select the radio button 'Small Classification' of 'TOE' for narrowing the range, check the check boxes 'Threat name' and 'Definition' of 'Threat' for display, input a keyword 'firewall' into the text box, and push the button 'Search.' Then, the threat names and definitions of threats which are assumed in the firewall category will be displayed.

Example 4: If users want to update the data;

Click the link of 'Update Function' in Fig. 2. If the users have no authority to update, they must get their account and password. Moreover, the users need to make a security specification document file for updating data according to STs or PPs. After that, click the link 'Update Function,' input the user name and password, push the button 'Reference,' select the document file, and push the 
button 'Upload.' The administrator will extract data from the uploaded file and update the data.

\section{Discussion}

We implemented the prototype user interface which has four functions. Function 1 supplies to easily and effectively retrieve the required data of design in secure system's specifications. Function 2 supplies to easily and effectively retrieve the required data of security criteria in ISO/IEC 15408. Function 3 supplies to freely retrieve the relationship of secure system design data. Function 4 supplies to update the data in ISEDS and to store secure system design data which are newly defined by users. These functions satisfy the requirements that we defined for ISEDS.

Furthermore, we evaluate the user interface from the viewpoint of usability engineering. There are two methods in usability evaluation, i.e., quantitative evaluation and qualitative evaluation. The quantitative evaluation is a questionnaire and the qualitative evaluation is heuristic evaluation or user tests [8]. Since it is difficult to perform questionnaires and user tests now, we evaluate the user interface with heuristic evaluation. The user interface satisfies five out of ten usability heuristics. ISEDS and its interface were implemented in a server which has suitable performance. Thus, the interface satisfies 'match between system and the real world,' one of the heuristic principles. Function 3 satisfies 'user control and freedom.' We unified the usage and display in every function. Therefore, the interface satisfies 'consistency and standards' and 'recognition rather than recall.' Since we prepared some explanations of the usage in the web pages, it also satisfies 'help and documentation.'

However, the interface does not satisfies the other heuristic principles 'visibility of system status,' 'error prevention,' 'flexibility and efficiency of use,' 'aesthetic and minimalist design,' and 'help users recognize, diagnose, and recover from errors.' For example, users may refer to what the other threats that are included in categories assuming a certain threat are. The users may also refer to what security functions are implemented in categories which require a certain criterion. The user interface cannot generate SQL sentences for such operations at once now. Users must have many intricate steps to execute such operations. We need to carefully discuss further improvement of the user interface for the efficiency and satisfaction.

\section{Concluding Remarks}

We have presented a web user interface of ISEDS. We designed, implemented, and evaluated the web user interface according to the requirements which are analyzed and defined from the viewpoint of software engineering, information security engineering, and usability engineering. Users can easily and effectively refer to secure system design data in ISEDS with web browsers. Therefore, ISEDS and its user interface can support design and development of secure information systems. 
Because the user interface has been evaluated only with qualitative method, we should also quantitatively evaluate it. Moreover, the structure of STs, PPs, security requirements, and the security functional requirements can easily, exactly and rigorously be expressed in XML. Therefore, we are improving ISEDS as a native XML database into which users can directly store the XML documents and are developing its web service. Moreover, ISEDS is deficient in some attributes of ISO/IEC 15408, STs, and PPs now, e.g., creation dates of STs or $\mathrm{PPs}$, evaluation assurance levels, and security assurance requirements defined in ISO/IEC 15408 Part 3. We are discussing whether or not these attributes are necessary.

\section{References}

1. Advanced Information Systems Engineering Laboratory, Saitama University.: ISEDS: Information Security Engineering Database System. http:// www.aise.ics.saitama-u.ac.jp/

2. Chen, P.: The Entity-Relationship Model - Toward a Unified View of Data. ACM Transactions on Database Systems (TODS), Volume 1, Issue 1, pp. 9-36 (1976)

3. International Software Benchmarking Standard Group.: Empirical Databases of Metrics Collected from Software Projects. http://www.isbsg.org/

4. ISO 9241-11 standard.: Ergonomic Requirements for Office Work with Visual Display Terminals - Part 11: Guidance on Usability (1998)

5. ISO/IEC 15408 standard.: Information Technology - Security Techniques - Evaluation Criteria for IT Security (1999)

6. Jiao, J. and Tseng, M.: A Requirement Management Database System for Product Definition. Journal of Integrated Manufacturing Systems, Vol. 10, No. 3, pp. 146-154 (1999)

7. Morimoto, S., Horie, D., and Cheng, J.: A Security Requirement Management Database Based on ISO/IEC 15408, in Computational Science and its Applications ICCSA 2006, International Conference, Glasgow, UK, May 8-11, 2006, Proceedings. Lecture Notes in Computer Science, Springer-Verlag, May (2006)

8. Nielsen, J. and Molich, R.: Heuristic Evaluation of User Interfaces. Proceedings of the SIGCHI conference on Human factors in computing systems: Empowering people, pp. 249-256, Seattle, WA, April (1990)

9. Software Engineering Institute.: Software Engineering Information Repository. http://seir.sei.cmu.edu/large 